\title{
ON THE UNIONS OF ASCENDING CHAINS OF DIRECT SUMS OF IDEALS OF $h$-LOCAL PRÜFER DOMAINS
}

\author{
J. E. MACÍAS-DÍAZ
}

\begin{abstract}
In this work, we investigate conditions under which unions of ascending chains of modules which are isomorphic to direct sums of ideals of an integral domain are again isomorphic to direct sums of ideals. We obtain generalizations of the Pontryagin-Hill theorems for modules which are direct sums of ideals of $h$-local Prüfer domains. Particularly, we prove that a torsion-free module over a Dedekind domain with a countable number of maximal ideals is isomorphic to a direct sum of ideals if it is the union of a countable ascending chain of pure submodules which are isomorphic to direct sums of ideals.
\end{abstract}

\section{IntRoduction}

In the last century, Lev Pontryagin and Paul Hill studied conditions under which torsion-free abelian groups are free. In their investigations, the concept of purity of subgroups was crucial. More precisely, a subgroup $H$ of an abelian group $G$ is pure if every equation of the form $k x=a \in H$, with $k \in \mathbb{Z}$, is solvable in $H$ whenever it is solvable in $G$. Equivalently, solubility in $G$ of each system of equations of the form

$$
\sum_{j=1}^{m} k_{i j} x_{j}=a_{i} \in H \quad(i=1, \ldots, n),
$$

with every $k_{i j} \in \mathbb{Z}$, implies its solubility in $H$.

In 1934, Pontryagin proved that a countable, torsion-free abelian group is free if and only if every finite rank, pure subgroup is free [11. Equivalently, every properly ascending chain of pure subgroups of the same finite rank is finite. From the proof of this result, it follows that a torsion-free abelian group $G$ is free if there exists an ascending chain

$$
0=G_{0} \leq G_{1} \leq \cdots \leq G_{n} \leq \ldots \quad(n<\omega)
$$

consisting of pure subgroups of $G$ whose union is equal to $G$, such that every $G_{n}$ is free and countable.

Later, in 1970, Hill established that, in order for an abelian group $G$ to be free, it is sufficient that it be the union of a countable ascending chain (2) of free, pure subgroups [7]. In other words, Hill proved that the condition of countability on the cardinality of the links $G_{n}$ in Pontryagin's theorem was superfluous. The proof of this theorem relies on some important facts about commutative groups, one of them being that subgroups of torsion-free abelian groups can be embedded in pure

Date: February 7, 2018.

2000 Mathematics Subject Classification. Primary 13C10, 13C05; Secondary 13F05, 16D40.

Key words and phrases. Pontryagin-Hill theorems, ascending chains of modules, direct sums of ideals, $h$-local Prüfer domains, balancedness of modules. 
subgroups of the same rank. Applications of these criteria may be actually found in a variety of algebraic results $[3,4,5,9$.

In view of the importance of the Pontryagin-Hill theorems in algebra, it is highly desirable to explore the possibility to generalize these criteria to more general scenarios. In this article, we generalize those results to modules which are isomorphic to direct sums of ideals of $h$-local Prüfer domains. Section 2 introduces the concept of balanced submodules and provides some useful criteria for balancedness. Section 3 serves as an introduction to $h$-local Prüfer domains and their properties, while Section 4 presents the most important theorems of this work.

\section{BALANCEDNESS}

Once and for all we declare that, throughout this work, $R$ will represent an integral domain unless stated otherwise. Modules are understood to be over $R$ when no other statement is done.

A submodule $N$ of an $R$-module $M$ is relatively divisible if the inclusion $N \cap r M \leq$ $r N$ holds, for every $r \in R$. Equivalently, solubility in $M$ of equations of the form $r x=a \in N$, with $r \in R$, implies their solubility in $N$. We say that $N$ is pure in $M$ if every finite system of equations

$$
\sum_{j=1}^{m} r_{i j} x_{j}=a_{i} \in N \quad(i=1, \ldots, n),
$$

with $r_{i j} \in R$, is solvable in $N$ whenever it is solvable in $M$. Under these circumstances, a short-exact sequence $0 \rightarrow N \rightarrow M \rightarrow Q \rightarrow 0$ is $R D$-exact (respectively, pure-exact) if $N$ is a relatively divisible (respectively, pure) submodule of $M$. Evidently, purity implies relative divisibility, and they both coincide for modules over Prüfer domains [12, that is, integral domains in which finitely generated ideals are projective. Moreover, Prüfer domains are the only integral domains for which relative divisibility and purity are equivalent 2 .

A submodule $A$ of the $R$-module $B$ is balanced if $B / A$ is torsion-free, and every rank 1, torsion-free $R$-module $J$ has the projective property with respect to the short-exact sequence $0 \rightarrow A \rightarrow B \rightarrow B / A \rightarrow 0$. In other words, for every homomorphism $\phi$ from $J$ into $B / A$, there exists a homomorphism $\psi$ from $J$ into $B$, which makes the following diagram commute:

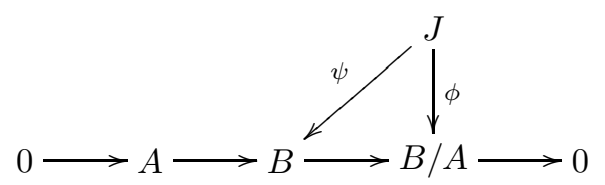

In this context, a short-exact sequence $0 \rightarrow A \rightarrow B \rightarrow C \rightarrow 0$ of $R$-modules, with $A$ balanced in $B$ and $C$ torsion free, is called balanced-exact. Clearly, direct sums of rank 1 modules have the projective property with respect to balancedexact sequences. For a list of relevant properties of relative divisibility, purity and balancedness of modules, we refer to [6].

Lemma 1. Let $L$ be a pure submodule of the torsion-free module $M$, with the property that $L$ is balanced in $N$, for every $L \leq N \leq M$ such that $N / L$ has rank 1 . Then, $L$ is balanced in $M$. 
Proof. Let $J$ be a rank 1, torsion-free module, and let $\phi$ be a homomorphism from $J$ into $M / L$. The image of $J$ under $\phi$ is a submodule $N / L$ of $M / L$ of rank at most 1. By hypothesis, there exists a homomorphism $\psi$ from $J$ into $L$, such that the following diagram with exact rows commutes:

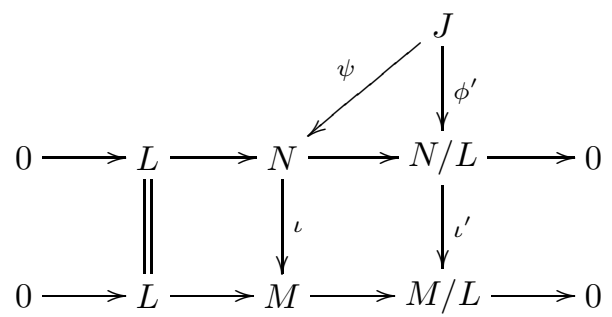

Here, the homomorphisms $\iota$ and $\iota^{\prime}$ are inclusions, and $\phi^{\prime}$ is the restriction of $\phi$ onto $N / L$. We conclude that $J$ is projective with respect to the sequence of the bottom row and, consequently, that $L$ is balanced in $M$.

We close this section with another criterion for balancedness.

Proposition 2. Consider the following commutative diagram with exact rows and torsion-free modules:

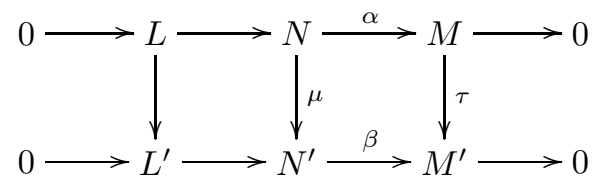

If there exists a homomorphism $\rho$ from $M^{\prime}$ to $M$, such that $\tau \rho=1$, and if the top row of (6) is balanced-exact, then the bottom row is also balanced-exact.

Proof. Let $\phi$ be a homomorphism from a rank 1, torsion-free module $J$ into $M^{\prime}$. Then, there exists a homomorphism $\sigma$ from $J$ into $N$, such that $\alpha \sigma=\rho \phi$. Clearly, the homomorphism $\psi=\mu \sigma$ has the property that $\beta \psi=\phi$, and we conclude that the bottom row is balanced-exact.

\section{3. $h$-LOCAL DOMAINS}

An $h$-local domain is an integral domain $R$ with the following properties:

(i) every nonzero prime ideal of $R$ is contained in exactly one maximal ideal, and

(ii) every nonzero element of $R$ is contained in all but a finite number of maximal ideals.

A valuation domain is an integral domain where ideals form a chain under inclusion; clearly, valuation domains have a unique maximal ideal. By a Dedekind domain we mean a hereditary domain, that is, a domain where all the ideals are projective. These two types of domains are examples of $h$-local Prüfer domain.

Given two ideals $I$ and $J$ of an integral domain $R$ with field of quotients $Q$, the residual of $I$ modulo $J$ is defined by $I: J=\{a \in Q: a J \leq I\}$. Indeed, Olberding proved [10] that a Prüfer domain $R$ is $h$-local if and only if $(J+K)$ : $I=(J: I)+(K: I)$, for any ideals $I, J$ and $K$ of $R$. With this characterization, he proves the following result, which generalizes the well-known fact that, over 
valuation domains and Dedekind domains, pure submodules of modules which are finite direct sums of ideals are direct summands isomorphic to direct sums of ideals.

Lemma 3 (Olberding [10]). Let $R$ be an h-local Prüfer domain, and let $A$ be a pure submodule of an $R$-module $B$ which is a finite direct sum of ideals of $R$. Then,

(i) $A$ is a summand of $B$, and

(ii) $A$ is isomorphic to a direct sum of ideals of $R$.

Lemma 4. Let $R$ be an h-local Prüfer domain. Every pure submodule of a module which is a countable direct sum of ideals of $R$ is isomorphic to a direct sum of ideals of $R$.

Proof. Let us assume that $M$ is the direct sum of the ideals $I_{n}$ of $R$, with $n<\omega$, and let $A$ be a pure submodule of $M$. Fix a maximal independent set $\left\{a_{n}: n \in \mathbb{Z}^{+}\right\}$ of $A$, and assume that, for some $n<\omega$, we have already constructed the finite ascending chain $0=A_{0} \leq A_{1} \leq \cdots \leq A_{n}$ of submodules of $A$, satisfying the following properties, for every $i<n$ :

(a) $A_{i+1}$ contains $\left\{a_{1}, \ldots, a_{i+1}\right\}$,

(b) $A_{i+1}$ is a finite rank, pure submodule of $A$, and

(c) $A_{i+1}=A_{i} \oplus B_{i}$, for some submodule $B_{i}$ of $A_{i+1}$ isomorphic to a direct sum of ideals of $R$.

Take a maximal independent set $Y_{n}$ of $A_{n}$, and fix a finite direct sum $N$ of ideals of $R$ in the decomposition of $M$, which contains the set $Y \cup\left\{a_{n+1}\right\}$. Clearly, the purification of this set has finite rank, is contained in $N$ and, by Lemma 3 , is a finite direct sum of ideals of $R$. Moreover, $A_{n+1}=A_{n} \oplus B_{n}$, for some submodule $B_{n}$ of $A_{n+1}$ isomorphic to a direct sum of ideals of $R$. By induction, $A$ is the union of the countable ascending chain

$$
0=A_{0} \leq A_{1} \leq \cdots \leq A_{n} \leq \ldots \quad(n<\omega) .
$$

We conclude that $A$ is isomorphic to the direct sum of the modules $B_{n}$, for $n<\omega$. Thus, $A$ is isomorphic to a direct sum of ideals of $R$.

Lemma 5. Let $R$ be an h-local Prüfer domain, let $M$ be a direct sum of ideals of $R$, and let $A$ be a pure submodule of $M$. If $A$ is the direct sum of countable rank submodules, then it is isomorphic to a direct sum of ideals of $R$.

Proof. Assume that $A$ is the direct sum of countable rank submodules $A_{\beta}$, where $\beta$ runs in a set of indexes $\Lambda$, and let $A$ be a pure submodule of $M=\oplus_{\alpha<\Omega} I_{\alpha}$, where every $I_{\alpha}$ is an ideal of $R$. Then, every $A_{\beta}$ is contained as a pure submodule in a countable direct sum of ideals of $R$ in the decomposition of $M$. By Lemma 4 , every $A_{\beta}$ is isomorphic to a direct sum of ideals of $R$ and, consequently, $A$ is likewise isomorphic to a direct sum of ideals of $R$.

For our next result, we employ the well-known theorem by Kaplansky which states that every direct summand of a direct sum of countable rank modules is also a direct sum of countable rank modules [8].

Theorem 6. Let $R$ be an h-local Prüfer domain. Every direct summand of a module which is a direct sum of ideals of $R$ is isomorphic to a direct sum of ideals of $R$. 
Proof. If $M$ is a direct sum of ideals of $R$, then it is a direct sum of countable rank submodules. If $A$ is a direct summand of $M$, then it is pure in $M$ and a direct sum of countable rank submodules. The conclusion is achieved now by means of Lemma 5.

\section{MAin Results}

Recall that a continuous, well-ordered, ascending chain of a module $M$ is an ascending chain

$$
0=A_{0} \leq A_{1} \leq \cdots \leq A_{\alpha} \leq A_{\alpha+1} \leq \ldots \quad(\alpha<\tau)
$$

of submodules of $M$ such that $A_{\alpha}=\bigcup_{\gamma<\alpha} A_{\gamma}$, for every limit ordinal $\alpha<\tau$.

Lemma 7. A torsion-free $R$-module $A$ is isomorphic to a direct sum of ideals of $R$ if it is the union of a continuous, well-ordered, ascending chain (8) of submodules, such that the following properties are satisfied, for every $\alpha<\tau$ :

(i) $A_{\alpha}$ is a balanced submodule of $A_{\alpha+1}$, and

(ii) $A_{\alpha+1} / A_{\alpha}$ is isomorphic to a direct sum of ideals of $R$.

Proof. For every $\alpha<\tau$, the balanced-exact sequence $0 \rightarrow A_{\alpha} \rightarrow A_{\alpha+1} \rightarrow A_{\alpha+1} / A_{\alpha} \rightarrow$ 0 splits. So, there exists a submodule $B_{\alpha}$ of $A_{\alpha+1}$ which is isomorphic to a direct sum of ideals of $R$, such that $A_{\alpha+1}=A_{\alpha} \oplus B_{\alpha}$. Then, $A$ is isomorphic to the direct sum of the modules $B_{\alpha}$ and, so, isomorphic to a direct sum of ideals of $R$.

The following is a generalization of Pontryagin's criterion of freeness to modules which are isomorphic to direct sums of ideals of an $h$-local Prüfer domain.

Theorem 8. Let $R$ be an h-local Prüfer domain. A countable rank, torsion-free module is isomorphic to a direct sum of ideals of $R$ if and only if every finite rank, pure submodule is isomorphic to a direct sum of ideals of $R$.

Proof. Let $M$ be a countable rank, torsion-free module, and assume that it is isomorphic to a direct sum of ideals of $R$. If $A$ is a finite rank, pure submodule of $M$, then it is contained in a finite direct sum $B$ of ideals in the decomposition of $M$. Lemma 3 implies that $A$ is isomorphic to a direct sum of ideals of $R$.

Conversely, let $\left\{a_{n}: n \in \mathbb{Z}^{+}\right\}$be a maximal independent set in $M$. For every positive integer $n$, let $A_{n}$ be the purification of $\left\{a_{1}, \ldots, a_{n}\right\}$ in $M$. Then, each $A_{n}$ is a finite rank, pure submodule of $M$ and, by hypothesis, isomorphic to a direct sum of ideals of $R$. In such a way, we construct a countable ascending chain (7) of pure submodules of $M$ which are isomorphic to finite direct sums of ideals of $R$. Clearly, $M$ is equal to the union of the links of (7). Moreover, Lemma 3 yields that, for every $n<\omega$, there exists a submodule $B_{n}$ of $A_{n+1}$ which is isomorphic to a finite direct sum of ideals of $R$, such that $A_{n+1}=A_{n} \oplus B_{n}$. Consequently, $M$ is isomorphic to a direct sum of ideals of $R$.

Theorem 9. Let $R$ be an h-local Prüfer domain. A torsion-free module $M$ is isomorphic to a direct sum of ideals of $R$ if it is the union of a countable ascending chain

$$
0=M_{0} \leq M_{1} \leq \cdots \leq M_{n} \leq \ldots \quad(n<\omega)
$$

of submodules, such that the following properties are satisfied, for every $n<\omega$ :

(i) $M_{n}$ is isomorphic to a direct sum of ideals of $R$, 
(ii) $M_{n}$ has countable rank, and

(iii) $M_{n}$ is pure in $M$.

Proof. Fix a countable maximal independent set $\left\{a_{n}: n \in \mathbb{Z}^{+}\right\}$of $M$, and assume that we have already constructed the links of a finite ascending chain

$$
0=A_{0} \leq A_{1} \leq \cdots \leq A_{n},
$$

for some $n<\omega$, such that the following properties are satisfied for every $i=1, \ldots, n$ :

(a) $A_{i}$ is isomorphic to a finite direct sum of ideals of $R$,

(b) $A_{i}$ contains $\left\{a_{1}, \ldots, a_{i}\right\}$, and

(c) $A_{i}$ is pure in $M$

Let $X_{n}$ be a maximal independent set of $A_{n}$, and let $k<\omega$ be such that $M_{k}$ contains both $X_{n}$ and $a_{n+1}$. Clearly, $A_{n}+a_{n+1} R$ is contained in a finite direct sum $A_{n+1}$ of ideals in the decomposition of $M_{k}$. Using induction, we construct a countable ascending chain (7) of finite rank, pure submodules of $M$ which are finite direct sums of ideals of $R$, and whose union is equal to $M$. Lemma 3 implies that $A_{n+1}=A_{n} \oplus B_{n}$, for every $n<\omega$, where $B_{n}$ is isomorphic to a finite direct sum of ideals of $R$. It follows that $M$ itself is isomorphic to a direct sum of ideals of $R$.

A $G\left(\aleph_{0}\right)$-family of an $R$-module $M$ is a family $\mathcal{B}$ consisting of submodules of $M$, with the following properties:

(i) $0, M \in \mathcal{B}$,

(ii) $\mathcal{B}$ is closed under unions of ascending chains of arbitrary lengths, and

(iii) for every $A_{0} \in \mathcal{B}$ and every countable set $H \subseteq M$, there exists $A \in \mathcal{B}$ containing $A_{0}$ and $H$, such that $A / A_{0}$ is countably generated.

Clearly, an intersection of a countable number of $G\left(\aleph_{0}\right)$-families of submodules of $M$ is again a $G\left(\aleph_{0}\right)$-family of submodules of $M$. The 'rank version' of this definition is called a $G\left(\aleph_{0}\right)^{\prime}$-family. More precisely, a $G\left(\aleph_{0}\right)^{\prime}$-family of $M$ is a family $\mathcal{B}$ of submodules of $M$, satisfying (i) and (ii) above, in addition to the property:

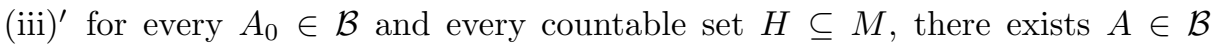
containing $A_{0}$ and $H$, such that $A / A_{0}$ has countable rank.

A $G\left(\aleph_{0}\right)$-family of submodules of $M$ is a tight system if, in addition, it satisfies:

(iv) for every $A \in \mathcal{B}, \operatorname{pd}_{R} A \leq 1$ and $\operatorname{pd}_{R}(M / A) \leq 1$.

It is worth noticing that every module has a $G\left(\aleph_{0}\right)$-family of submodules, namely, the collection of all its submodules. However, not every module has a $G\left(\aleph_{0}\right)$-family consisting of pure submodules. Nevertheless, Bazzoni and Fuchs proved [1] that every torsion-free module of projective dimension at most equal to 1 over a valuation domain has a tight system consisting of pure submodules.

It is important to recall that valuation domains have a unique maximal ideal. Moreover, localizations of Prüfer domains are again Prüfer domains and, particularly, localizations of Prüfer domains at prime ideals are valuation domains. As a consequence, every torsion-free module of projective dimension at most 1 over a Prüfer domain with a countable number of maximal ideals has a $G\left(\aleph_{0}\right)$-family consisting of pure submodules. Indeed, let $M$ be a torsion-free $R$-module of projective dimension at most 1 , where $R$ is a Prüfer domain with a countable number of maximal ideals. For each maximal ideal $P$ of $R$, let $\mathcal{B}_{P}^{\prime}$ be a $G\left(\aleph_{0}\right)$-family of pure submodules of the localization of $M$ with respect to $P$, and let $\mathcal{B}_{P}=\left\{A \cap M: A \in \mathcal{B}_{P}^{\prime}\right\}$. The desired family is the intersection of all the families $\mathcal{B}_{P}$. 
We prove next a generalization of Hill's theorem to modules which are isomorphic to direct sums of ideals of $h$-local Prüfer domains.

Theorem 10. Let $R$ be an h-local Prüfer domain. A torsion-free module $M$ is isomorphic to a direct sum of ideals of $R$ if it is the union of a countable ascending chain (9) of submodules, such that the following properties are satisfied, for every $n<\omega$ :

(i) $M_{n}$ is isomorphic to a direct sum of ideals of $R$,

(ii) $M_{n+1} / M_{n}$ has a $G\left(\aleph_{0}\right)$-family $\mathcal{C}_{n}$ of pure submodules, and

(iii) $M_{n}$ is pure in $M$.

For each $n<\omega$, the module $M_{n}$ can be written as the direct sum of $R$-modules $I_{\alpha}^{n}$, with $\alpha$ in some set of indexes $\Omega_{n}$, each of which is isomorphic to an ideal of $R$. The collection $\mathcal{B}_{n}$ of submodules of $M$ of the form $\oplus_{\alpha \in \Lambda} I_{\alpha}^{n}$, for some $\Lambda \subseteq \Omega_{n}$, is a $G\left(\aleph_{0}\right)^{\prime}$-family of pure submodules of $M_{n}$, for every $n<\omega$. Moreover, the collection

$$
\mathcal{B}_{n}^{\prime}=\left\{A \in \mathcal{B}_{n}: \frac{\left(A+M_{j}\right) \cap M_{j+1}}{M_{j}} \in \mathcal{C}_{j}, \text { for every } j<n\right\} \quad(n<\omega)
$$

is a $G\left(\aleph_{0}\right)^{\prime}$-family of pure submodules of $M_{n}$. Furthermore, the class

$$
\mathcal{B}=\left\{A \leq M: A \cap M_{n} \in \mathcal{B}_{n}^{\prime} \text {, for every } n<\omega\right\}
$$

is a $G\left(\aleph_{0}\right)^{\prime}$-family of pure submodules of $M$, such that for every $n<\omega$ and every $A \in \mathcal{B}_{n}$, the module $A+M_{n}$ is pure in $M$.

Lemma 11. For every $A \in \mathcal{B}$, finite rank, pure submodules of $M / A$ are isomorphic to direct sums of ideals of $R$.

Proof. Let $D$ be a submodule of $M$ containing $A$, such that $D / A$ is a finite rank, pure submodule of $M / A$, and choose a countable set of representatives $S \subseteq D$ of a maximal independent system of $D$ modulo $A$. Let $k<\omega$ be an index such that $S \subseteq M_{k}$. Since $A+M_{k}$ and $D$ are pure in $M$, then $A+\left(D \cap M_{k}\right)=D \cap\left(A+M_{k}\right)$ is a pure submodule between $A$ and $D$ which contains $S$, so that $D=A+\left(D \cap M_{k}\right)$. Now, $A \cap M_{k}$ belongs to $\mathcal{B}_{k}^{\prime}$ and, consequently, it is a direct summand of $M_{k}$, say, $M_{k}=\left(A \cap M_{k}\right) \oplus B$, for some submodule $B$ of $M_{k}$. Therefore, $D \cap M_{k}=$ $\left(A \cap M_{k}\right) \oplus(B \cap D)$.

Being isomorphic to a submodule of a finite rank module, $C=B \cap D$ is a finite rank, pure submodule in the module $M_{k}$ which, in turn, is isomorphic to a direct sum of ideals. So, $C$ itself is isomorphic to a direct sum of ideals by Lemma 3 . Moreover, since

$$
D=A+\left(D \cap M_{k}\right)=\left(A+\left(A \cap M_{k}\right)\right) \oplus C=A \oplus C,
$$

$D / A$ is isomorphic to a direct sum of ideals.

The following result is a consequence of the proof of Lemma 11

Lemma 12. Every $A \in \mathcal{B}$ is a balanced submodule of $M$.

Proof. Since $A$ is a direct summand of every submodule $D$ of $M$ for which $D / A$ has rank 1 , then $A$ is balanced in $D$. The conclusion of this result follows now from Lemma 1 .

We are now in a position to prove our generalization of Hill's theorem to modules which are isomorphic to direct sums of ideals of $h$-local Prüfer domains. 
Proof of Theorem 10. Let $\alpha$ be any nonzero ordinal, and assume that we have already constructed the links of the continuous, well-ordered, ascending chain

$$
0=A_{0} \leq A_{1} \leq \cdots \leq A_{\gamma} \leq A_{\gamma+1} \leq \ldots \quad(\gamma<\alpha)
$$

of modules of $\mathcal{B}$, such that $A_{\gamma+1} / A_{\gamma}$ is isomorphic to a direct sum of ideals of $R$, for every $\gamma<\alpha$. If $\alpha$ is a limit ordinal, we let $A_{\alpha}$ be the union of the links of chain (14). Otherwise, $\alpha$ is the successor of an ordinal number $\beta$. If there exists $x \in M \backslash A_{\beta}$, then there exists $A_{\alpha} \in \mathcal{B}$ containing both $x$ and $A_{\beta}$, such that $A_{\alpha} / A_{\beta}$ has countable rank. Finite rank, pure submodules of $A_{\alpha} / A_{\beta}$ are isomorphic to direct sums of ideals of $R$ by Lemma 11, thus $A_{\alpha} / A_{\beta}$ itself is a direct sum of ideals of $R$ by Lemma 5 . In such way, we construct a continuous, well-ordered, ascending chain (8) satisfying the hypothesis of Lemma 7 . We conclude that $M$ is isomorphic to a direct sum of ideals of $R$.

Since Dedekind domains are $h$-local Prüfer domains for which every ideal is projective, we have the following obvious improvement of Theorem 10 .

Corollary 13. Let $R$ be a Dedekind domain with a countable number of maximal ideals. The torsion-free module $M$ is isomorphic to a direct sum of ideals of $R$ if it is the union of a countable ascending chain (9) of submodules, such that the following are satisfied, for every $n<\omega$ :

(i) $M_{n}$ is isomorphic to a direct sum of ideals of $R$, and

(ii) $M_{n}$ is pure in $M$.

Proof. For every $n<\omega, M_{n+1} / M_{n}$ is a torsion-free module of projective dimension less than or equal to 1 . The observation preceding Theorem 10 implies now that every factor module of (9) admits a $G\left(\aleph_{0}\right)$-family of pure submodules. By Theorem 10. $M$ is isomorphic to a direct sum of ideals of $R$.

It is worth noticing that the conclusion of Corollary 13 is reached if, in particular, the links $M_{n}$ are free modules. Moreover, it is easy to check that if $M$ is a torsionfree module over an integral domain $R$ which is the union of the countable ascending chain (9) of projective, pure submodules, then there exists a chain

$$
0=F_{0} \leq F_{1} \leq \cdots \leq F_{n} \leq \ldots \quad(n<\omega)
$$

consisting of free $R$-modules, such that:

(a) every $F_{n}$ is pure in $F_{n+1}$,

(b) every $F_{n}$ contains $M_{n}$ as a direct summand, say, $F_{n}=M_{n} \oplus K_{n}$,

(c) $M$ is a direct summand of $F=\bigcup_{n<\omega} F_{n}$, and

(d) $\left\{K_{n}\right\}_{n<\omega}$ may be chosen to form an ascending chain under inclusion.

The proof of the next result is now straight-forward.

Corollary 14. Let $R$ be a Dedekind domain with a countable number of maximal ideals. The torsion-free module $M$ is projective if it is the union of a countable ascending chain (9) of projective, pure submodules.

Acknowledgments. The author wishes to acknowledge the guidance of Prof. László Fuchs at every stage of this investigation. Also, he wishes to thank the anonymous reviewer for her/his kind comments, which led to improve the quality of this work. The results presented here are part of research project PIM10-01 at the Universidad Autónoma de Aguascalientes. 


\section{REFERENCES}

[1] S. Bazzoni and L. Fuchs. On modules of finite projective dimension over valuation domains. In Proceedings of the Conference on Abelian Groups and Modules in Udine, volume 287 of CISM Courses and Lectures, pages 361-371. Springer, 1984.

[2] H. Cartan and S. Eilenberg. Homological Algebra. Princeton Landmarks in Mathematics. Princeton University Press, Princeton, New Jersey, 1st edition, 1999.

[3] E. F. Cornelius. A generalization of separable groups. Pac. J. Math., 39(3):603-613, 1971.

[4] P. C. Eklof. Whitehead's problem is undecidable. Amer. Math. Month., 83(10):775-788, 1976.

[5] P. C. Eklof and S. Shelah. A non-reflexive Whitehead group. J. Pure Appl. Alg., 156(23):199-214, 2001.

[6] L. Fuchs and L. Salce. Modules over non-Noetherian Domains, volume 84 of Mathematical Surveys and Monographs. American Mathematical Society, Providence, Rhode Island, 1st edition, 2001.

[7] P. Hill. On the freeness of abelian groups: a generalization of Pontryagin's theorem. Bull. Amer. Math. Soc., 76(5):1118-1120, 1970.

[8] I. Kaplansky. Projective modules. Annals of Math., 68(2):372-377, 1958.

[9] A. H. Mekler and S. Shelah. Every coseparable group may be free. Israel J. Math., 81(12):161-178, 1993.

[10] B. Olberding. Prüfer domains and pure submodules of direct sums of ideals. Mathematika, 46(2):425-432, 1999.

[11] L. Pontryagin. The theory of topological commutative groups. Annals of Math., 35(2):361$388,1934$.

[12] R. B. Warfield. Purity and algebraic compactness of modules. Pac. J. Math., 28:699-719, 1969 .

Departamento de Matemáticas y Física, Universidad Autónoma de Aguascalientes, Avenida Universidad 940, Ciudad Universitaria, Aguascalientes, Ags. 20100, Mexico

E-mail address: jemacias@correo.uaa.mx 